\title{
USO DE CONTRACETIVOS EM ALUNOS DO ENSINO SUPERIOR
}

\section{USE OF CONTRACEPTIVES IN STUDENTS FROM HIGHER EDUCATION USO DE ANTICONCEPTIVOS EN ALUMNOS DE EDUCACIÓN SUPERIOR}

\author{
Duarte Loio (a23830@alunos.ipb.pt)* \\ Eva Festa (a23833@alunos.ipb.pt)* \\ Carlos Ferreira (a23826@alunos.ipb.pt)* \\ Diana Soares (a23829@alunos.ipb.pt)* \\ António Fernandes (toze@ipb.pt)* \\ Luís Nascimento (luis.miguel@ipb.pt) ${ }^{\star * *}$
}

\section{RESUMO}

É sabido que, durante a adolescência e a juventude, acontece um despertar gradual e paulatino para a sexualidade e nem sempre existe a devida proteção, através do uso de métodos contracetivos. Também é verdade que não existe um método ideal de contraceção, o que nos levou a indicar os quatro grandes tipos de métodos contracetivos, designadamente, os métodos naturais/comportamentais, os métodos da barreira contracetiva, os métodos de contraceção hormonal e os métodos de contraceção cirúrgica ou esterilização.

Realizou-se um estudo de caráter quantitativo, transversal, comparativo (teste de Mann-WhitneyWilcoxon; teste de Kruskal-Wallis) e correlacional (teste de Spearman), através de um questionário administrado diretamente, a 365 alunos do Instituto Politécnico de Bragança (IPB), com idades compreendidas entre os 17 e os 32 anos, tendo como objetivo avaliar o conhecimento e as atitudes dos alunos face ao uso de métodos contracetivos.

Os métodos contracetivos mais utilizados pelos alunos questionados são o preservativo e a pilula. No entanto, os resultados apontam para algum desconhecimento do tema em causa, o que nos leva a concluir pela inexistência de boas práticas no uso de contracetivos por parte dos alunos do IPB e, porventura, generalizando, da falta de uma intervenção estruturada no âmbito da Educação Sexual.

Palavras-chave: Conhecimento, Atitudes, Estudantes, Métodos Contracetivos, Ensino Superior.

\begin{abstract}
It is known that, during adolescence and youth, comes a progressive and slow awakening for sexuality and there is not always an adequate protection through the use of contraceptive methods. It is also true, that there is no ideal contraception method, which led us to indicate the four major types of contraceptive methods, namely: natural/behavioral, barrier contraceptive, hormonal contraception and surgical methods of contraception or sterilization. We conducted a quantitative, transversal, analytical, comparative (MannWhitney-Wilcoxon test; Kruskal-Wallis test) and correlational (Spearman test) study through a questionnaire administrated directly to the students from the Polytecnic Institute of Bragança (IPB), with ages between 17 and 32 years. The objectives of this study were to evaluate their knowledge of the use of contraceptives as well as their attitudes towards the use of contraceptives. The contraceptive methods most used by students surveyed were condoms and the pill. However, the results point to some
\end{abstract}




\section{Eqitania \\ s c i e $\Omega$ c i a}

ignorance about this subject, which leads us to conclude about the absence of good practices in the use of contraceptives among students of the Polytechnic Institute of Bragança and, perhaps, more generally, the lack of any structured intervention in the area of Sex Education.

Keywords: Knowledge, Attitudes, Students, Contraceptive Methods, Higher Education.

\section{RESUMEN}

Se sabe que, durante la adolescencia y la juventud, llega un despertar gradual y progresivo de la sexualidad y, a veces, no hay una protección adecuada a través del uso de métodos anticonceptivos. También es cierto, que no existe un método ideal de anticonceptivos, lo que nos llevó a indicar los cuatro tipos principales de métodos anticonceptivos, a saber: los métodos naturales de conducta, los métodos anticonceptivos de barrera, los métodos anticonceptivos hormonales y los métodos quirúrgicos de anticoncepción o la esterilización.

Se realizó un estudio cuantitativo, transversal, analítico, comparativo (prueba de Mann-Whitney-Wilcoxon, prueba de Kruskal-Wallis) y de correlación (prueba de Spearman) mediante un cuestionario directamente administrado a los estudiantes del Instituto Politécnico de Bragança (IPB), con edades entre 17 y 32 años, teniendo como tema el conocimiento y sus prácticas sobre el uso de anticonceptivos.

Los métodos anticonceptivos más utilizados por los estudiantes fueron de condones y las pildoras. Sin embargo, los resultados muestran una cierta ignorancia sobre el tema, lo que nos lleva a concluir por la ausencia de buenas prácticas en el uso de anticonceptivos entre los estudiantes de IPB y, tal vez, más en general, la falta de una intervención estructurada bajo la Educación Sexual.

Palabras-clave: Conocimientos, Actitudes, Estudiantes, Métodos Anticonceptivos, Educación Superior.

* Técnicos de Farmácia licenciados pela Escola Superior de Saúde do Instituto Politécnico de Bragança.

** Vice-coordenador do Departamento de Ciências Sociais e Exatas da Escola Superior Agrária do Instituto Politécnico de Bragança. Membro efetivo do Centro de Estudos Transdisciplinares para o Desenvolvimento da Universidade de Trásos-Montes e Alto Douro.

*** Membro do Departamento das Tecnologias da Saúde da Escola Superior de Saúde do Instituto Politécnico de Bragança 


\section{Egitania \\ $s$ c i e $\cap$ c i a}

\section{INTRODUÇÃO}

A Organização Mundial de Saúde (1989) definiu "juventude" como o conjunto de pessoas com idades entre os 15 e os 24 anos, considerando "pessoas jovens " a faixa etária entre os 10 e os 24 anos e especificando a adolescência como subgrupo desses jovens, situando-os entre os 10 e os 19 anos de idade (Moura, Borges \& Rocha, 2008). Em Portugal, segundo o Instituto Nacional de Estatística (2012), o conceito de jovem em abrange as pessoas no escalão etário dos 15 aos 29 anos.

Por razões práticas, vamos utilizar o termo "jovem(ns)" para este case study, apesar de alguns questionários terem sido preenchidos por alguns alunos cujas idades estão compreendidas entre os 17 e os 32 anos.

O processo de transição dos jovens para a vida adulta alterou-se muito nas sociedades ocidentais durante o século XIX, considerado por muitos especialistas das diversas ciências como o século das grandes revoluções.

A faixa etária considerada é uma fase especial e complexa, um período de transição em que o jovem procura o seu lugar de autoafirmação na sociedade, ou a procura dela, perante os adultos; enfim, uma época estruturante da sua mentalidade, que pode, isso facto, determinar, positiva ou negativamente, o seu próprio futuro. Assim, não olvidemos que se trata de uma etapa da vida, marcada pela ocorrência de um complexo processo de crescimento e desenvolvimento biopsicossocial, com inúmeras alterações e transformações físicas, psicológicas e sociais. Estamos perante uma etapa profundamente influenciada por fatores pessoais, familiares e socioculturais. (Mendonça e Araúijo, 2009).

As alterações físicas assumem um carácter complexo. Alguns absorvem facilmente essas alterações, enquanto a maioria necessita de mais algum tempo para se adaptar, "visto que estas modificações possuem uma relação direta com a identidade psicológica e sexual do indivíduo", como sublinham Mendonça e Araújo (2009).

Além das transformações fisiológicas e anatómicas, tenhamos em consideração também todo o conjunto de alterações emocionais e comportamentais que estruturam a personalidade dos jovens. Daí a sua natural repercussão no âmbito da saúde sexual e reprodutiva, arrastando obviamente alguma ou demasiada vulnerabilidade perante os riscos a que se expõem, nomeadamente, a possibilidade de uma gravidez indesejada e precoce, além da exposição às doenças sexualmente transmissíveis. No entanto, apesar disso, ao longo do século XX, algumas iniciativas foram tomadas relativamente às questões sociais e entre elas a da sexualidade do adolescente que, segundo Moura, Borges e Rocha (2008), ganha importância com os escritos de Freud e de Stanley Hall em 1904. Aliás, os estudos mostram que, cada vez mais, o início da atividade sexual acontece na adolescência. (Alves e Lopes, 2008)

Assim, podemos corroborar a afirmação de Bozon e Heilborn (2006) de que a sexualidade tem aspetos que são fundamentais para a construção da identidade pessoal. Para os homens, sublinham os mesmos autores, a iniciação sexual é considerada prova de masculinidade 


\section{Eqitania}

$s$ c i e $\Omega$ c i a

heterossexual, podendo ocorrer com qualquer parceira e o mais cedo possível. Para as mulheres, a iniciação sexual é tradicionalmente interpretada como parte de um relacionamento.

Nesta faixa etária, existe geralmente um despertar gradual e paulatino da sexualidade. No entanto, há casos em que esse despertar manifesta-se ex abrupto, talvez porque tenha passado a ser conduzida pela constante busca do prazer, tornando a sexualidade feminina não inteiramente ligada à procriação (devido à presença cada vez mais forte das mulheres nas mais diversas atividades sociais), e daí o aumento da possibilidade de riscos, apesar de estarmos perante uma geração cada vez mais informada sobre a sexualidade (Pedro, 2003)

Segundo Souza e Osório citados por Berger (1999), a sexualidade faz parte da vida e o seu equilíbrio depende da estabilidade emocional do indivíduo. E, sublinham que, entre os seres humanos, a sexualidade não diz respeito apenas à procriação, concluindo que a sexualidade inclui, necessariamente, os sentimentos de amor e prazer. No entanto, de acordo com Pirotta e Schor (2004), ao negligenciarem a prática da contraceção e da prevenção às doenças sexualmente transmissíveis, adolescentes e jovens expõem-se ao HIV e a outras doenças sexualmente transmissíveis e a gravidezes não planeadas.

É, neste contexto, que assume particular relevo o conhecimento e a escolha de um método anticoncecional, o mais eficaz possível. Porém, os jovens, com o impulso frenético de iniciar a atividade sexual, descuram a devida proteção, conduzindo assim a gravidezes indesejáveis e expondo-se às doenças sexualmente transmissíveis.

Todavia, a escolha do método anticoncecional não deixa de se tornar tão complexa quanto a imaturidade e a instabilidade do jovem. A falta de estruturação do seu temperamento e, sobretudo, o desconhecimento dos métodos disponíveis, os riscos de uma gravidez, a vivência e o relacionamento afetivo no âmbito da sexualidade, são fatores envolvidos na escolha do método mais adequado (Madureira e Weber, 2011). Além disso, é fundamental que os métodos anticoncecionais sejam conhecidos no âmbito do seu funcionamento, das vantagens e desvantagens e do nível de segurança.

Assim sendo, e tendo por base estas premissas, consideramos pertinente e relevante a investigação das condutas e práticas dos alunos do IPB relativamente ao uso de contracetivos. Os objetivos desta investigação passam por avaliar o grau de conhecimento acerca dos métodos contracetivos; verificar se o uso destes métodos é ou não adequado; verificar se existe relação entre o conhecimento de métodos contracetivos e o seu uso adequado; e, ainda, determinar qual o método contracetivo mais utilizado pelos alunos do IPB.

O artigo estrutura-se em seis secções, nomeadamente, a introdução, o enquadramento teórico, a metodologia, os resultados, a discussão dos resultados e, por fim, a conclusão. Na primeira secção, definem-se os objetivos e faz-se uma abordagem geral ao tema. Na segunda, faz-se a revisão da literatura de forma a enquadrar teoricamente o tema em estudo. A terceira diz respeito à metodologia usada nesta investigação, designadamente, participantes, material e procedimento. Na quarta secção, apresentam-se os resultados relativos às diferentes variáveis 


\section{Egitania}

$s$ c i e $\Omega$ c i a

em estudo. Na quinta, faz-se a discussão dos resultados e a consequente comparação com a literatura. E, por último, na conclusão, apresentam-se as considerações finais.

\section{ENQUADRAMENTO TEÓRICO}

O campo da saúde continua a atrair a atenção dos jovens alunos e a acalentar toda e qualquer oportunidade que lhes permita erguer e conduzir fileiras importantes dentro do seu raio de ação como futuros profissionais. Devem, por isso, questionar e desafiar temas da mais diversa índole, quebrando tabus e procurando o conhecimento teórico, bem como a realidade empírica, dentro do universo que os rodeia.

Um desses temas é, sem dúvida, a contraceção. Na atualidade, a formação individualista da sociedade fez com que o despertar para a sexualidade seja visto como um processo de afirmação individual e prazer da juventude. A desinformação, segundo Moura, Gomes, Rodrigues, e Oliveira (2011:321), é consequência de "conceitos equivocados, carregados de tabus, oriundos de colegas e amigos que não tiveram acesso à educação sexual", sublinhando que, por isso, a desinformação torna-se um círculo vicioso, difícil de quebrar. Tal facto, justifica este trabalho de investigação sobre as práticas no uso de contracetivos por parte dos alunos do IPB.

A contraceção pode, desde já, ser definida muito simplesmente como a prevenção voluntária da gravidez, permitindo afirmar que a eficácia da maioria das estratégias contracetivas depende, principalmente, da motivação dos jovens que as usa e sublinhar que nenhuma opção contracetiva é 100 por cento eficaz, fácil de usar e isenta de efeitos secundários (Norvitz e Schorge, 2003).

Aliás, a contraceção não é um percurso linear, havendo oscilação de métodos de baixa e de alta eficácia e muitas vezes prevalecendo a não utilização de qualquer método (Bataglião e Mamede, 2011). A questão passa, sobretudo, pela não consistência no uso dos métodos contracetivos, pois certamente ocorrem alternâncias de métodos conforme as relações sexuais se vão estabilizando ou quando novos relacionamentos se iniciam. Por isso, os jovens, tal como os adultos, devem possuir um conhecimento completo de todos eles e das boas práticas no seu uso, desde as respetivas indicações e contraindicações terapêuticas, até aos efeitos colaterais e eficácia teórica e prática. Aliás, o facto de se oferecerem opções de escolha dos diversos métodos aos jovens provoca segurança e, sem dúvida, uma melhor utilização dos mesmos, o que resulta, certamente, numa vida sexual livre de riscos e com maior satisfação (Mendonça e Araújo, 2009).

Efetivamente, existem inúmeros métodos contracetivos, designadamente, os métodos naturais/comportamentais, os métodos da barreira contracetiva, os métodos de contraceção hormonal e os métodos de contraceção cirúrgica ou esterilização. 


\section{Egitania}

s c i e $\Omega$ c i a

\section{OS MÉTODOS NATURAIS/COMPORTAMENTAIS}

Os métodos comportamentais são, segundo Lopes, Silva, Moraes, Aquino, Américo e Pinheiro (2010), os menos utilizados e os menos conhecidos pela população que tem mais dificuldades na sua utilização. Estes métodos, de acordo com Enciclopédia Médica llustrada para a Saúde da Familia (1983), baseiam-se todos na fisiologia normal da mulher, exigindo um grande conhecimento relativamente ao próprio corpo, nomeadamente na observação atenta das características do seu ciclo menstrual; por isso, são designados "naturais". Na prática, são métodos que procuram determinar quais os dias fecundos através de sintomas e sinais. Incluem-se neste quadro o método de Ogino, também apelidado do calendário, o método da temperatura basal, o método Billings, igualmente conhecido por método do muco e/ou de ovulação e, ainda, o método do coito interrompido.

O método de Ogino (calendário) é um método pouco fiável de contraceção, sobretudo para jovens e mulheres com ciclos curtos e/ou muito irregulares. Quanto ao método da temperatura, convém referir que, durante a ovulação, a temperatura da mulher sofre um ligeiro aumento, normalmente entre $0,2^{\circ}$ e $0,5^{\circ} \mathrm{C}$, que se mantém até ao aparecimento da menstruação. Nestes dias de temperatura mais elevada a mulher é estéril. No caso do método Billings, durante a ovulação e nos dias que a precedem surgem numerosas alterações biológicas significativas, nomeadamente a ocorrência de um muco, que é uma substância gelatinosa, produzida pelo cérvix (o colo do útero). Nos dias em que há muco, a mulher é fecunda; nos dias em que não há muco, é estéril. O método do coito interrompido é conhecido como o método de contraceção mais antigo, consistindo em retirar o pénis da vagina antes da ejaculação. É um método com inconvenientes para o homem porque pode sentir inibição, devido à retirada antes da ejaculação e para a mulher por não atingir o clímax e criar sentimentos de frustração.

Estamos, por isso, perante métodos que apresentam baixa eficácia, alteram o comportamento do casal, necessitam de aprendizagem e não protegem contra doenças sexualmente transmissíveis.

\section{OS MÉTODOS DE BARREIRA CONTRACETIVA}

Segundo Norwitz e Schorge (2003) estes métodos impedem a penetração do esperma vivo no útero, quer matando os espermatozoides, quer obstruindo-lhes a passagem. Integram este campo o preservativo, o diafragma, os espermicidas e o dispositivo intrauterino (DIU).

O preservativo evita a deposição de sémen na vagina, é facilmente acessível e, usado com o devido cuidado, previne a disseminação de doenças sexualmente transmissíveis. Profissionais de saúde e educadores em geral devem incentivar o uso do preservativo, como um importante mecanismo de contraceção. Segundo Santos, Rebouças, Boery, Boery e Silva (2009:119), "um estudo realizado nos Estados Unidos demonstrou que o correto e sistemático uso de preservativos em todas as relações sexuais apresenta uma eficácia estimada em 90-95\% na prevenção da transmissão do HIV", e "sugerem uma relação linear entre a frequência do uso de preservativos e a redução do risco de transmissão". 


\section{Egitania}

s c i e $\cap$ c i a

O diafragma impede a passagem do esperma para o canal cervical, mas é conveniente que seja retirado algumas horas após o coito. Daí que a sua aplicação exija uma determinada aprendizagem e o seu tamanho deva adaptar-se ao da vagina.

Os espermicidas são contracetivos químicos destinados a serem profundamente introduzidos na vagina imediatamente antes do coito. Podem ser usados singularmente, mas devido à sua hipotética falta de eficácia, devem ser utilizados juntamente com preservativos ou diafragmas.

O DIU é um contracetivo muito utilizado em todo o mundo, sendo um dispositivo geralmente feito de cobre, colocado dentro do útero e da tuba uterina. Deve ser inserido por um(a) médico(a) ou enfermeiro(a) especializados para o efeito, devido às reações adversas que pode provocar, nomeadamente, a tendência para infeções na região pélvica durante o primeiro ano de utilização, as quais podem reduzir a fertilidade. O DIU pode ser sujeito a uma remoção precoce se surgir menorragia e /ou dismenorreia.

\section{OS MÉTODOS DE CONTRACEÇÃO HORMONAL}

Estes métodos são constituídos por hormonas sintéticas que atuam no centro regulador do ciclo menstrual, provocando um estado de não ovulação. São bastante eficazes e possuem uma taxa de gravidez muito baixa. Abrangem este painel, fundamentalmente, os contracetivos orais, os contracetivos injetáveis e a contraceção de emergência ou do dia seguinte.

Existem dois tipos fundamentais de contracetivos orais: as pilulas mistas, que contêm progesterona e hormonas estrogéneas, e as pilulas progestativas, que contêm apenas progesterona.

Nas pilulas mistas, o estrogénio atua impedindo a libertação de óvulos dos ovários; a progesterona coagula o muco do cérvix, ou colo uterino, dificultando a penetração dos espermatozoides, e altera as características do revestimento uterino, tornando-o desfavorável à implantação do óvulo fecundado. A pilula mista não deve ser tomada por mulheres com doença hepática ou que tenham tido embolias, tromboses ou outros problemas circulatórios; é uma pilula que também agrava as enxaquecas. No entanto, quando tomada regularmente, é um dos contracetivos mais seguros. As perdas sanguíneas correspondentes à menstruação são regulares, menos abundantes e normalmente indolores, mas o seu uso não é aconselhado durante a amamentação, pois pode diminuir o fluxo de leite. Estas pílulas constituem o método de contraceção mais popular e mais utilizado. Como exemplo, em 2005/2006, estas pilulas destacaram-se, em Portugal continental, com uma percentagem de 65,9\%, seguindo-se o preservativo, com 13,4\% e o DIU com 8,8\%. Nos ditos anos, nas Regiões Autónomas dos Açores e da Madeira, foi igualmente o método mais utilizado com 57,7\% e 61,3\%, respetivamente (Instituto Nacional de Estatística, 2007)

As pilulas progestativas, de progesterona ou micropílulas previnem a gravidez atuando perifericamente de modo a diminuir a função dos ovidutos e a reduzir a produção de muco cervical. São, ligeiramente, menos seguras do que as pilulas mistas e podem provocar hemorragias irregulares. 


\section{Egitania}

$\mathrm{s}$ c i e $\Omega$ c i a

Os dois tipos de pllulas indicados anteriormente existem também sob a forma de contracetivos injetáveis, possuindo um efeito de longa duração. Os efeitos secundários são mais acentuados, com um ciclo menstrual frequentemente perturbado e exigindo, por isso, um controle médico muito cuidadoso.

Quanto à contraceção de emergência ou pilula do dia seguinte, trata-se da obtenção de efeitos abortivos em casos de probabilidade de fecundação, quer dificultando a implantação do óvulo fertilizado no útero, quer impedindo o seu desenvolvimento, caso a implantação se tenha efetivado, com a consequente morte do embrião. Estes efeitos podem ser obtidos ingerindo uma dose elevada da pilula na manhã seguinte à prática do ato sexual. Porém, a sua ingestão pode provocar náuseas e vómitos. Este método é indicado somente em casos de emergência, como o próprio nome revela, e não como método contracetivo de uso rotineiro (Ribeiro e Fernandes, 2014). Na opinião de Borges, Fujimori, Hoga e Contin (2010:817), "existe uma variedade de situações em que é recomendado, [caso] da ocorrência de violência sexual, na rutura acidental do preservativo ou diafragma, na expulsão do DIU, [ ], no atraso menstrual de mais de duas semanas". A utilização da contraceção de emergência tem aumentado proporcionalmente ao uso cada vez maior dos métodos contracetivos mais populares e mais utilizados. (Norwitz e Schorge, 2003)

\section{OS MÉTODOS DE CONTRACEÇÃO CIRÚRGICA}

Estes métodos, de acordo com a Enciclopédia Médica llustrada para a Saúde da Familia (1983), designados também por esterilização, implicam uma intervenção cirúrgica que pode ser realizada tanto no homem como na mulher e representa uma contraceção de carácter permanente.

A esterilização masculina, a vasectomia, é uma pequena intervenção cirúrgica que consiste em cortar e laquear os canais deferentes, através dos quais o esperma passa dos testículos para o pénis. A vasectomia não afeta, em geral, o desejo nem a prática sexual.

A esterilização feminina implica a laqueação das trompas de Falópio, que conduzem o óvulo do ovário para o útero.

Um método de esterilização definitiva é a histerectomia, ou seja, a remoção cirúrgica do útero. Trata-se de uma grande intervenção cirúrgica com indicações médicas bem definidas.

\section{METODOLOGIA}

Para um nível de confiança de 95\% e tendo em conta a população objeto deste estudo, ou seja, a população de estudantes matriculados no IPB no ano letivo de 2012/2013, foi calculada a dimensão da amostra através do Sample Size Calculator. Assim, a partir de uma população de cerca de 6000 estudantes que frequentam o IPB (exceto Polo Mirandela) foram administrados diretamente, 368 inquéritos de forma aleatória, sendo três invalidados devido a preenchimento incorreto. Desta forma, a amostra em estudo é constituída por 365 alunos. 


\section{Egitania}

$s$ c i e $\Omega$ c i a

Foram excluídos desta investigação alunos do Instituto Politécnico de Bragança do Polo de Mirandela devido ao critério geográfico.

A amostra desta investigação foi recolhida nos meses de outubro e novembro de 2012 nas Escolas do IPB situadas nesta cidade, nomeadamente, Escola Superior de Saúde (ESSa), Escola Superior Agrária (ESA), Escola Superior de Educação (ESE) e Escola Superior de Tecnologias e Gestão (ESTIG).

O método de recolha de dados nesta investigação foi a inquirição e a técnica o questionário de autopreenchimento. O questionário foi estruturado em três partes. A primeira parte incluía questões acerca dos atributos individuais nomeadamente, o género, a idade, o estado civil e o método contracetivo utilizado com mais frequência e, ainda, questões relativas à academia como a escola, o curso e o ano frequentado. A segunda parte pretendia avaliar o conhecimento dos alunos no que diz respeito aos métodos contracetivos. Estes conhecimentos foram avaliados pelo Contraceptive Knowlegde Inventory (Caldeira, Galhardas, Nobre, e Ramiro, 2005), escala de autoavaliação, constituída por 9 itens que avaliam conhecimentos biológicos, modos de atuação e crenças face aos métodos contracetivos. Cada item tem 5 opções de resposta, mas apenas uma é a correta. Os resultados obtidos variam entre 0 e 9, com o valor mais alto como indicador de maior conhecimento face aos métodos contracetivos. A terceira e última parte avalia as atitudes dos alunos face aos métodos contracetivos. As atitudes e prevenção do risco foram avaliadas pela Contraceptive Attitude Scale (Caldeira, Galhardas, Nobre e Ramiro, 2005), escala de autoavaliação, constituída por 11 itens que avaliam as atitudes face ao parceiro e atitudes face a si próprio no uso de métodos contracetivos. Os resultados obtidos podem variar em 11 e 55 pontos com o valor mais alto como indicador de atitudes positivas para a utilização de contraceção-prevenção de risco.

O presente estudo é de carácter transversal, dado que os questionários foram administrados aos estudantes num só momento; analítico pois recorre-se a testes estatísticos, e, observacional visto que o estudo se limita a analisar o comportamento dos inquiridos face ao uso de métodos contracetivos. Esta investigação é de carácter quantitativo e tem como variáveis independentes a idade, o género, a escola e o estado civil. As variáveis dependentes são o conhecimento, atitudes face à prevenção do risco e os métodos contracetivos.

Após a autorização da Presidência da Instituição, pediu-se a colaboração dos estudantes para o preenchimento do questionário durante o qual os investigadores estavam presentes para o esclarecimento de dúvidas que pudessem surgir. Aquando da entrega dos questionários, os alunos foram informados da confidencialidade dos dados e dos objetivos da investigação.

Depois de recolhidos todos os questionários estes foram numerados e inseridos no programa Statistical Package for Social Sciences (versão 20.0 para o Windows), seguindo-se o tratamento estatístico. De modo a descrever o grupo de alunos questionado, utilizou-se estatística univariada para caracterizar a amostra, nomeadamente, medidas de tendência central (média) e medidas de dispersão (mínimo, máximo e desvio padrão) para as variáveis quantitativas. No caso das variáveis qualitativas, medidas numa escala nominal, calcularam-se frequências 


\section{Egitania}

$s$ c i e $\Omega$ c i a

relativas e absolutas (Pestana e Gageiro, 2005; Maroco, 2007) que serão apresentadas sob a forma de tabelas de frequências e de gráficos.

Posteriormente, o estudo analítico dos dados permitiu comparar os conhecimentos e as atitudes tendo em consideração fatores como o género, a idade, a escola e o estado civil. Para efetuar estas comparações recorreu-se a testes não-paramétricos uma vez que sempre que se testaram as condições de aplicação destes testes, nomeadamente, a normalidade dos dados com recurso ao teste de Kolmorov-Smirnov com a correção de Lilliefords ( $N \geq 30$ ) ou ao teste de Shapiro-Wilk ( $<30)$; e, a igualdade de variâncias com recurso ao teste de Levene, pelo menos uma destas condições era violada.

O teste de Kolmorov-Smirnov é, segundo Maroco (2007), um teste não-paramétrico de aderência à Distribuição Normal e testa a hipótese nula da variável em estudo seguir essa distribuição com uma determinada média ( $\mu$ ) e uma determinada variância (62), ou seja, HO: X N ( $\mu$; 62), contra a hipótese alternativa da variável em estudo não seguir a Distribuição Normal, isto é, $H 1: X \sim / N(\mu ; 62)$.

O teste de Levene permite, segundo e Maroco (2007), testar a hipótese nula das variâncias dos grupos serem homogéneas, ou seja, HO: $621=622=\quad=62 \mathrm{k}$, contra a hipótese alternativa de existir pelos menos um par de variâncias diferentes ( $H 1$ : $\exists i$, j: $62 \mathrm{i} \neq \mathbf{6}$ 2j).

Para realizar a comparação tendo em conta o género e o estado civil usou-se o teste de MannWhitney-Wilcoxon (teste não-paramétrico) em alternativa ao teste T-Student (teste paramétrico) para amostras independentes. Segundo Maroco (2007), o teste de Mann-Whitney-Wilcoxon permite testar a hipótese nula da igualdade de medianas, ou seja, permite testar a inexistência de diferenças significativas entre as medianas $(\eta)$ das duas amostras comparadas ( $\mathrm{HO}: \eta 1=$ $\eta$ 2) contra a hipótese alternativa das medianas serem diferentes ( $H 1: \eta 1 \neq \eta 2)$.

Para realizar a comparação tendo em conta a escola e a classe etária, ou seja, entre mais de duas (K) amostras independentes usou-se o teste de Kruskal-Wallis (teste não paramétrico) em alternativa ao teste da ANOVA One Way (teste paramétrico). Segundo Pestana e Gageiro (2005) e Maroco (2007), o teste de Kruskal-Wallis permite testar a hipótese nula da igualdade de medianas ( $\mathrm{HO}: \eta 1=\eta 2=\quad=\eta \mathrm{k})$ contra a hipótese alternativa de existir pelo menos um par de medianas diferentes $(H 1: \exists i, j: \eta i \neq \eta j)$.

O estudo analítico dos dados permitiu, também, estudar a corelação entre o nível de conhecimento e as atitudes dos alunos face aos métodos contracetivos. Como ambas as variáveis são qualitativas, medidas numa escala ordinal, utilizou-se o teste de correlação ordinal de Spearman pois permite testar a hipótese nula das variáveis não estarem correlacionadas contra a hipótese alternativa das variáveis estarem correlacionadas. Trata-se de um coeficiente que, segundo Pestana e Gageiro (2005) e Maroco (2007), varia entre -1 (correlação inversa perfeita) e 1 (correlação direta perfeita).

Na execução dos testes estatísticos foi utilizado o nível de significância ( $\alpha$ ) de 5\% por, em investigação científica, ser razoável a implementação de testes com um grau de confiança de 
95\%. Nesta situação, o Erro do Tipo I (rejeitar a hipótese nula quando esta é verdadeira) é de 5\%. Sempre que possível, tentou aumentar-se o grau de confiança para 99\% a que corresponde um $\alpha$ de $1 \%$.

\section{RESULTADOS}

A idade dos inquiridos variou entre os 17 e os 32 anos, sendo a média de idades de 21,02 $( \pm 2,47)$.

Dos 365 alunos inquiridos, 55,3\% eram do género feminino e 44,7\% eram do género masculino (Tabela 1).

Quanto ao estado civil, existe uma prevalência de alunos solteiros $(97,3 \%)$ e apenas uma pequena percentagem de casados $(1,9 \%)$, de divorciados $(0,5 \%)$ e de viúvos $(0,3 \%)$, tal como pode ver-se na Tabela 1.

A distribuição da amostra por escola é a seguinte: 121 alunos da Escola Superior de Tecnologia e Gestão (33,2\%); 102 alunos da Escola Superior de Saúde (27,9\%); 102 alunos da Escola Superior de Educação (27,9\%); e 40 alunos da Escola Superior Agrária (11\%), como pode verse na Tabela 1.

$O$ ano mais frequentado pelos inquiridos era o $2^{\circ}$ ano com um registo de 33,8\%; de seguida o $1^{\circ}$ ano com 31,5\% e o $3^{a}$ ano com 30,4\%. Os restantes frequentavam o $4^{a}$ ano $(2,0 \%)$ e cursos de Mestrado (2,3\%), como pode ver-se na Tabela 1

\begin{tabular}{|c|c|c|c|}
\hline \multirow[t]{2}{*}{ Variável } & \multirow[t]{2}{*}{ Categoria } & \multicolumn{2}{|c|}{ Frequências } \\
\hline & & $\begin{array}{c}\text { Absolutas } \\
(\mathrm{N})\end{array}$ & $\begin{array}{c}\text { Relativas } \\
\text { (\%) }\end{array}$ \\
\hline \multirow[t]{2}{*}{ Género } & Masculino & 163 & 44,7 \\
\hline & Feminino & 202 & 55,3 \\
\hline \multirow[t]{4}{*}{ Estado Civil } & Solteiro & 354 & 97,3 \\
\hline & Casado & 7 & 1,9 \\
\hline & Divorciado & 2 & 0,5 \\
\hline & Viúvo & 1 & 0,3 \\
\hline \multirow[t]{4}{*}{ Escola } & Escola Superior de Saúde (ESSa) & 102 & 27,9 \\
\hline & Escola Superior Agrária (ESA) & 40 & 11,0 \\
\hline & Escola Superior de Tecnologia e Gestão (ESTiG) & 121 & 33,2 \\
\hline & Escola Superior de Educação (ESE) & 102 & 27,9 \\
\hline \multirow{5}{*}{$\begin{array}{l}\text { Ano } \\
\text { Frequentado }\end{array}$} & $1^{\circ}$ Ano & 110 & 31,5 \\
\hline & $2^{\circ}$ Ano & 118 & 33,8 \\
\hline & $3^{\circ} \mathrm{Ano}$ & 106 & 30,4 \\
\hline & $4^{\circ}$ Ano & 7 & 2,0 \\
\hline & Mestrado & 8 & 2,3 \\
\hline
\end{tabular}

Tabela 1 - Caraterização da amostra $(n=365)$ 


\section{Eqitania}

$s$ c i e $\Omega$ c i a

Quanto aos métodos contracetivos, verifica-se que 178 dos alunos inquiridos utilizam o preservativo (49,7\%), 75 utilizam a pilula (20,9\%) e, igualmente, 75 utilizam a pilula e o preservativo conjuntamente (20,9\%), sendo estes os métodos mais utilizados pelos alunos inquiridos (Figura 1).

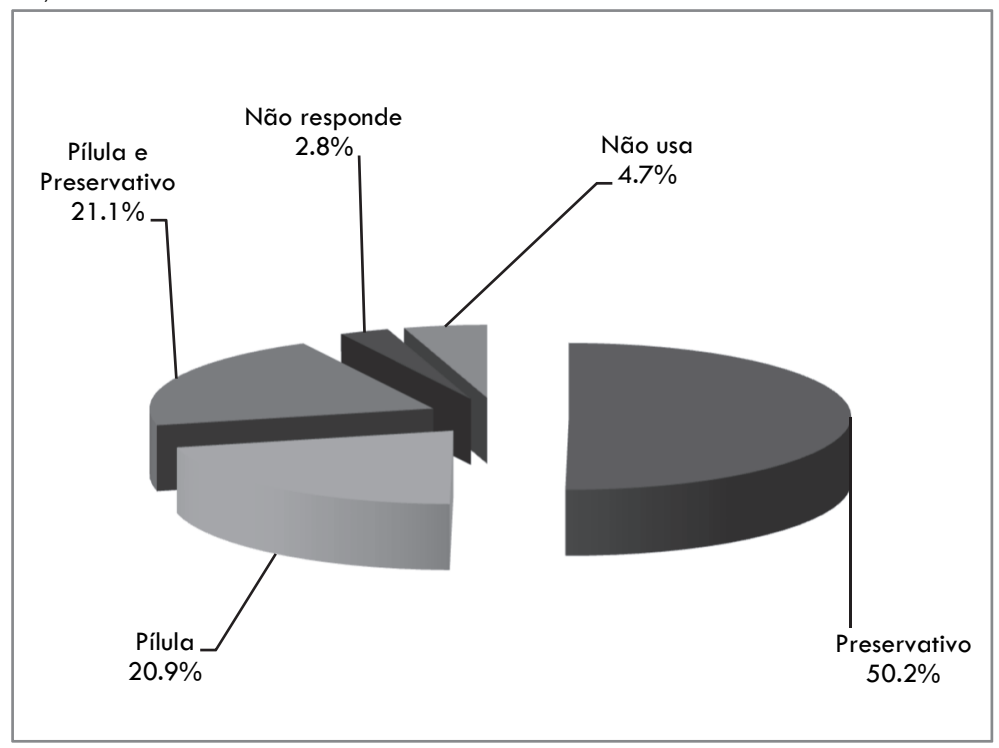

Figura 1 - Métodos contracetivos usados pelos alunos do IPB $(n=358)$

Quanto aos restantes inquiridos, alguns não responderam à pergunta (2,8\%), outros afirmaram que não usavam $(4,7 \%)$ e alguns indicaram que usam outro tipo de método contracetivo (0,9\%) como, por exemplo, o anel vaginal, contracetivos injetáveis ou a contraceção de emergência (Figura 1).

O nível médio do conhecimento sobre métodos contracetivos medidos numa escala de 0 a 9 pontos foi de apenas 4,6 ( $\pm 2,053)$. Para verificar se o género era um fator diferenciador do conhecimento sobre métodos contracetivos usou-se, como foi referido na metodologia, o teste de Mann-Whitney-Wilcoxon. Este teste forneceu um p-value de 0,000, rejeitando-se a hipótese nula. Por essa razão, é possível afirmar com um grau de confiança de 99\%, que o conhecimento sobre métodos contracetivos varia consoante o género. Para além disso, como pode ver-se na Tabela 2, foi possível verificar que esse conhecimento é mais elevado nos indivíduos do género feminino (Mean Rank = 217,97).

O teste de Mann-Whitney-Wilcoxon permitiu verificar que o estado civil não era um fator diferenciador do conhecimento sobre métodos contracetivos ( $p$-value = 0,581), tal como pode ver-se na Tabela 2.

Para verificar se a escola era um fator diferenciador do conhecimento acerca dos métodos contracetivos, recorreu-se ao teste de Kruskal-Walis. Este teste forneceu um p-value de 0,000 que conduz à rejeição da hipótese nula permitindo afirmar, com um grau de confiança de 99\%, que o nível de conhecimento sobre métodos contracetivos é diferente, tendo em consideração o fator escola (Tabela 2). Para identificar as diferenças referidas, utilizou-se a comparação múltipla de medianas, recorrendo-se para o efeito ao teste de Mann-Whitney-Wilcoxon. 0 


\section{Eqitania}

$s$ c i e $\Omega$ c i a

output deste teste permitiu verificar que o nível de conhecimento era superior na Escola Superior de Saúde (Mean Rank = 233,81).

Para verificar se o conhecimento sobre os métodos contracetivos era influenciado pela classe etária, recorreu-se ao teste de Kruskal-Walis. A Tabela 2 mostra que este teste forneceu um pvalue de 0,016, o que implica rejeitar a hipótese nula. Assim sendo, com um grau de confiança de 95\%, conclui-se que o nível de conhecimento sobre métodos contracetivos difere tendo em conta a classe etária. Através do teste de Mann-Whitney-Wilcoxon procedeu-se à comparação múltipla de medianas de modo a identificar as diferenças referidas. O output deste teste permitiu verificar que o conhecimento é superior nos indivíduos da classe etária dos 19 a 22 anos (Mean Rank = 190,22).

\begin{tabular}{|c|c|c|c|c|}
\hline Fator & Categorias & $\mathrm{N}$ & $\begin{array}{l}\text { Mean } \\
\text { rank }\end{array}$ & $p$-value \\
\hline \multirow{2}{*}{$\begin{array}{l}\text { Género } \\
(\mathrm{N}=365)\end{array}$} & Masculino & 163 & 139,66 & \multirow{2}{*}{$0,000^{*}$} \\
\hline & Feminino & 202 & 217,97 & \\
\hline \multirow{2}{*}{$\begin{array}{l}\text { Estado civil } \\
(\mathrm{N}=364)\end{array}$} & Solteiro & 354 & 183,01 & \multirow{2}{*}{0,581} \\
\hline & Outro & 10 & 164,55 & \\
\hline \multirow{4}{*}{$\begin{array}{l}\text { Escola } \\
(\mathrm{N}=365)\end{array}$} & Escola Superior Agrária (ESA) & 40 & 163,16 & \multirow{4}{*}{$0,000^{*}$} \\
\hline & Escola Superior de Educação (ESE) & 102 & 169,30 & \\
\hline & Escola Superior de Saúde (ESSa) & 102 & 233,81 & \\
\hline & Escola Superior de Tecnologia e Gestão (ESTiG) & 121 & 133,99 & \\
\hline \multirow{3}{*}{$\begin{array}{l}\text { Idade } \\
\qquad(\mathrm{N}=364)\end{array}$} & Inferior a 19 anos & 28 & 189,82 & \multirow{3}{*}{$0,016^{\star \star}$} \\
\hline & De 19 a 22 anos & 266 & 190,22 & \\
\hline & Superior a 22 anos & 70 & 150,24 & \\
\hline
\end{tabular}

Relativamente às atitudes face ao parceiro e face a si próprio no que diz respeito ao uso de contracetivos, pode-se afirmar que os alunos do IPB apresentaram atitudes bastante positivas, com uma média de 35,5 $( \pm 4,494)$ numa escala que variava entre 11 e 55 pontos. Verificou-se, ainda, que as atitudes face ao uso de contracetivos são mais adequadas no grupo dos indivíduos do género feminino, tal como mostra a Tabela 3. O output do teste de Mann-WhitneyWilcoxon forneceu um p-value $=0,008$, que sendo inferior ao nível de significância de 0,01 implica a rejeição da hipótese nula. Por essa razão, conclui-se que as atitudes dos alunos face ao uso de métodos contracetivos são, estatisticamente, diferentes e melhores nos indivíduos do género feminino (Mean Rank = 187,12). 


\section{Egitania}

$s$ c i e $\Omega$ c i a

A Tabela 3 revela a ausência de diferenças nas atitudes face aos métodos contracetivos segundo o estado civil. De fato, o teste de Mann-Whitney-Wilcoxon forneceu um p-value de 0,694, o que implica não rejeitar a hipótese nula. Desta forma, conclui-se, ao nível de significância de 1\%, que não existem diferenças significativas nas atitudes dos alunos do IPB no que diz respeito ao uso dos métodos contracetivos tendo em conta o fator estado civil.

A Tabela 3 coloca em evidência a existência de diferenças nas atitudes face aos métodos contracetivos quando o fator escola é tido em consideração. Efetivamente, o output do teste de Kruskal-Wallis permitiu verificar a existência de diferenças significativas nas atitudes dos alunos uma vez que o p-value $(0,002)$ é inferior ao nível de significância $(0,01)$. Para identificar essas diferenças utilizou-se, como referido anteriormente, à comparação múltipla de medianas usando, para o efeito, o teste de Mann-Whittney-Wilcoxon. O output deste teste permitiu verificar que as atitudes dos alunos da ESSa (Mean Rank = 198,43) e da ESA (Mean Rank = 203,29) são, estatisticamente, diferentes e mais adequadas que as atitudes dos alunos da ESTIG e da ESE.

\begin{tabular}{|c|c|c|c|c|}
\hline Fator & Categorias & $\mathrm{N}$ & $\begin{array}{c}\text { Mean } \\
\text { rank }\end{array}$ & $p$-value \\
\hline \multirow{2}{*}{$\begin{array}{l}\text { Género } \\
(\mathrm{N}=348)\end{array}$} & Masculino & 152 & 158,23 & \multirow{2}{*}{$0,008^{*}$} \\
\hline & Feminino & 196 & 187,12 & \\
\hline \multirow{2}{*}{$\begin{array}{l}\text { Estado civil } \\
(\mathrm{N}=347)\end{array}$} & Solteiro & 337 & 174,36 & \multirow{2}{*}{0,694} \\
\hline & Outro & 10 & 161,75 & \\
\hline \multirow{4}{*}{$\begin{array}{l}\text { Escola } \\
(\mathrm{N}=348)\end{array}$} & Escola Superior Agrária (ESA) & 36 & 203,29 & \multirow{4}{*}{$0,002^{*}$} \\
\hline & Escola Superior de Educação (ESE) & 98 & 157,71 & \\
\hline & Escola Superior de Saúde (ESSa) & 102 & 198,43 & \\
\hline & Escola Superior de Tecnologia e Gestão (ESTiG) & 112 & 158,14 & \\
\hline \multirow{3}{*}{$\begin{array}{l}\text { Idade } \\
(\mathrm{N}=348)\end{array}$} & Inferior a 19 anos & 27 & 202,04 & \multirow{3}{*}{0,304} \\
\hline & De 19 a 22 anos & 253 & 172,46 & \\
\hline & Superior a 22 anos & 57 & 168,51 & \\
\hline
\end{tabular}

A Tabela 3 revela, ainda, a inexistência de diferenças nas atitudes face aos métodos contracetivos quando a idade é tida em consideração. De fato, como o p-value $(0,304)$ fornecido pelo teste de Kruskal-Wallis é superior ao nível de significância $(0,05)$, não se pode 


\section{Egitania}

$s$ c i e $\cap$ c i a

rejeitar a hipótese nula. Por essa razão, conclui-se que a idade não é um fator diferenciar no que diz respeito às atitudes dos alunos face ao uso dos métodos contracetivos.

Como foi referido na metodologia, para verificar se o conhecimento e o uso adequado dos métodos contracetivos estão correlacionados utilizou-se o teste da correlação ordinal de Spearman. Este teste forneceu um p-value de 0,033 que, sendo inferior ao nível de significância de 0,05, conduziu à rejeição da hipótese nula. Conclui-se, com um grau de confiança de 95\%, que o conhecimento acerca dos métodos contracetivos está correlacionado com as atitudes dos alunos face ao uso desses mesmos métodos. O valor do coeficiente de correlação é de 0,115 mostrando que existe uma correlação direta entre as duas variáveis. No entanto, a correlação é fraca, pois o valor encontra-se próximo de zero.

\section{DISCUSSÃO}

O presente estudo teve como objetivo principal avaliar o nível de conhecimento relativamente aos métodos contracetivos bem como avaliar as atitudes em relação aos mesmos tendo em conta as hipóteses apresentadas na metodologia. Os resultados demonstraram que as raparigas têm mais conhecimentos sobre os métodos contracetivos que os rapazes; as raparigas têm atitudes mais positivas face à prevenção do risco do que os rapazes; o nível de conhecimento está correlacionado com as atitudes dos jovens, e o método contracetivo mais utilizado pelos jovens é o preservativo.

No que diz respeito ao conhecimento relativo aos métodos contracetivos, este estudo demonstrou que o nível médio do conhecimento sobre métodos contracetivos medido numa escala de 0 a 9 pontos é de apenas 4,6 pontos, tal como sucedeu no estudo de Ribeiro e Fernandes (2014). Já, no que diz respeito às atitudes face ao risco, pode-se afirmar que os alunos do IPB não aceitam correr riscos no que toca à sexualidade, apresentando, no geral, atitudes bastante positivas, com uma média de 35,5 numa escala que varia entre 11 e 55 pontos. Estes resultados são consistentes como os obtidos por Ribeiro e Fernandes (2009) num estudo realizado na mesma instituição no qual verificaram que os jovens estudantes do IPB demonstraram saber que o preservativo é o único meio de proteção das doenças sexualmente transmissíveis. No entanto, nesse mesmo estudo que, dos $76 \%$ respondentes que já tinham tido relações sexuais, 40\% tiveram-nas sob o efeito de álcool, 8,1\% sob o efeito de drogas e 3,6\% nunca usaram preservativo.

Relativamente aos conhecimentos quando comparados entre géneros, a análise estatística mostrou diferenças significativas, confirmando que as raparigas possuem maior conhecimento ao nível da contraceção do que os rapazes. O mesmo se pode afirmar em relação às atitudes face à prevenção do risco, no qual se verificou que as raparigas são mais prudentes que os rapazes. Estes resultados são consistentes com a literatura, (Belo \& Silva, 2004; Almeida, Duarte, André \& Lalanda, 2004; Ribeiro e Fernandes, 2014). 


\section{Egitania}

$s$ c i e $\cap$ c i a

No que diz respeito ao estudo da relação entre o nível de conhecimento e as atitudes face à prevenção do risco, verificou-se a existência de uma correlação fraca e direta do conhecimento com as atitudes. Resultados esses que vão ao encontro dos obtidos por Roque (2001).

No que toca ao método contracetivo mais utilizado pelos alunos do IPB comprovou-se a preferência pelo uso do preservativo, uma vez que é o método mais utilizado pelos jovens. Este resultado é consistente com os obtidos por Magalhães, Carrilho e Leite (2001) e Ribeiro e Fernandes (2014).

\section{CONCLUSÃO}

Os problemas relacionados com a saúde dos seres humanos não podem ser resolvidos pelo ceticismo, cujos horizontes são limitados por realidades práticas.

Sabemos, também, que o conhecimento e a informação da sexualidade entre os jovens têm sido limitados por algumas sociedades. No entanto, devido ao desenvolvimento tecnológico relativo aos métodos contracetivos e aos avanços no âmbito da saúde sexual, a informação disponibilizada é uma das melhores formas de aderir a um programa de prevenção. Daí que a orientação contracetiva deva consistir num trabalho educativo baseado na informação e conhecimento corretos e credíveis sobre a saúde sexual.

Esta vertente da saúde passa, incondicionalmente, pela Educação Sexual, que deveria ser ministrada nas faixas etárias da adolescência e da juventude. Estas são fases especiais e complexas da vida, marcadas por um processo de autoafirmação, crescimento e desenvolvimento biopsicossocial. Este processo de autoafirmação verifica-se também no despertar gradual e paulatino da sexualidade que inclui, essencialmente nesta fase, o sentimento de prazer e não de procriação, como nos parece óbvio. Por isso, é naturalmente descurada a devida proteção, o que pode provocar uma gravidez indesejável ou a exposição a uma doença sexualmente transmissível.

Ora, as conclusões que se podem retirar dos resultados obtidos permitem verificar que os alunos do IPB não aceitam correr riscos no que toca à sexualidade. Este facto pode derivar do facto de já terem beneficiado de alguma educação sexual, mesmo que esta tenha sido meramente informal.

Denota-se, através dos resultados, que a responsabilidade contracetiva está mais direcionada para as raparigas, talvez pelo facto do risco da conceção e das doenças sexualmente transmissíveis. No entanto, Vilar (2003) alerta para a existência de mensagens sexuais contínuas que associam a contraceção à mulher, o que justifica uma intervenção estruturada de Educação Sexual no sentido de promover a igualdade de géneros sobre os conhecimentos acerca dos métodos contracetivos.

A amostra estudada pode ser considerada bastante representativa da população escolar, neste caso, alunos do IPB. Aquando da recolha de dados pode ter ocorrido algum "enviesamento" de 


\section{Eqitania \\ $s$ c i e $\Omega$ c i a}

informação relativamente ao comportamento contracetivo dos inquiridos, dado que as questões constituem um assunto de natureza íntima que pode trazer algum constrangimento ou desconfiança em relação ao sigilo das informações em causa. No entanto, de modo a minimizar esta limitação, foram aplicados questionários anónimos de participação voluntária tendo o compromisso dos investigadores, de carácter verbal e escrito, da confidencialidade das informações obtidas.

Como foi referido, os resultados do presente estudo reforçam a necessidade de investir na Educação Sexual da comunidade em geral, mas principalmente da população adolescente e jovem, promovendo o acesso à informação necessária para a correta utilização dos métodos contracetivos.

Espera-se que esta investigação possa ter contribuído para a ampliação e compreensão do nível de conhecimentos e das atitudes dos jovens estudantes do IPB e, sobretudo, para a necessidade de sensibilizar a população para a promoção da educação para a saúde e para a sexualidade, nesta etapa tão importante da vida do ser humano.

\section{REFERÊNCIAS}

Almeida, A.; Duarte, V.; André, l.; Lalanda, P. (2004); Fecundidade e contracepção: Percursos de Saúde Reprodutiva das Mulheres PORTUGUESAS; LISBOA: INSTITUTO DE CIÊNCIAS SOCIAIS DA UNIVERSIDADE DE LISBOA.

AlVES, A. \& LOPES, M. (2008); CONHECIMENTO, ATITUDE E PRÁTICA DO uSO dE PÍlULA E PRESERVATIVO ENTRE ADOLESCENTES UNIVERSITÁRIOS; REVISTA BRASILEIRA DE ENFERMAGEM; 61:1;11-17.

ALVES, C. \& BRANDÃO, R. (2008), VULNERABILIDADE NO USO DE MÉTODOS CONTRACEPTIVOS ENTRE ADOLESCENTES E JOVENS: INTERSECÇÕES ENTRE políticas públicas e atenção à Saúde; Rio de Janeiro: Instituto Fernandes figueiras e Instituto de Estudos em Saúde Colectiva. FACULDADE de MEDICINA, UniVersidade Federal.

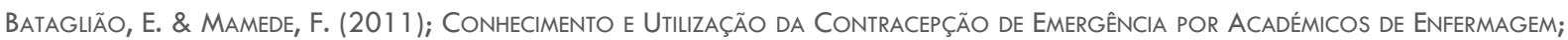
Escola AnNa Nery Revista de Enfermagem; 15: 2; 284-290.

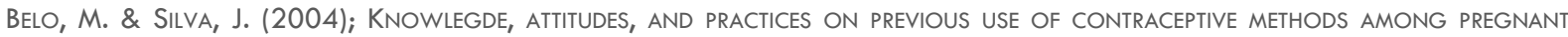
teENAGers; REVISTA DE SAÚde PúBlICA, 38: 4; 479-487.

Berger, H (1999); O perfil do educador Gaúcho em relação À SeXualidade; ReV. Brasileira de SeXualidade Humana; $10: 1 ; 89-118$.

BORGES, A.; FuJIMORI, E.; HOGA, L. \& CONTIN, M. (2010); PrátICAS CONTRACEPTIVAS ENTRE JOVENS UNIVERSITÁRIOS: O USO dA ANTICONCEPÇÃO DE EMERGÊNCIA; CAD. SAÚde PúbLICA; 26: 4; 816-826.

Bozon, M. \& Hellborn, M. (2006); INICIAÇÃo À SEXUALIdADE: MOdOS de SOCIALIZAÇÃO, INTERACÇÕes E TRAJeCtóRIAS INDIVIDUAIS. IN: M. L. HeILBORN ET AL. (ORGS.). O APRENDIZADO DA SEXUALIDADE: REPRODUÇÃO E TRAJETÓRIAS SOCIAIS DE JOVENS BRASILEIROS. RIO DE JANEIRO: FIOCRUZ.

Caldeira, C.; Galhardas, M.; Nobre, M. \& Ramiro, L. (2005); Conhecimentos e Atitudes face aos Métodos Contraceptivos em Jovens ADULTOS; MANUSCRITO NÃO PUBLICADO.

EnCiclopédia Médica Ilustrada para a Saúde da Família (1984). Lisboa: Selecções do Reader's Digest.

Instituto Nacional de Estatística (2007); INQuérito Nacional de Saúde 2005/2006; lisboa: Instituto Nacional de Estatística e INSTITUTO NACIONAL DE SAÚDE DR. RICARDO JORGE. 


\section{Eqitania \\ s c i e $\Omega$ c i a}

instituto Nacional de Estatística (2012); Censos 2011 ; Lisboa: Instituto Nacional de Estatística.

Lopes, E.; Silva, S.; Moraes, M.; Aquino, P.; Américo, C.; Pinheiro, A. (2010); Conhecimento de enfermeiros sobre métodos Contraceptivos no ConteXto do programa Saúde da família; ENFermería Global; 20 ; 1 -9.

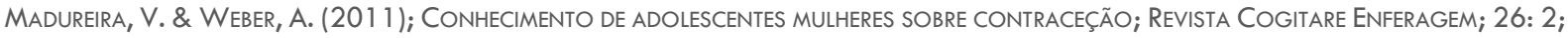
333-339.

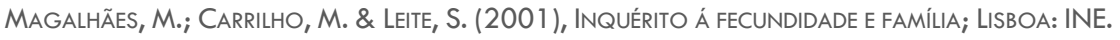

Maroco, J. (2007); ANÁLISE Estatística COM UTILIZAÇÃo do SPSS; LISBOA: EdIçÕES SílAbO.

Mendonça, R. \& ARaúJo, T. (2009); MÉtodos ContraCetivos: A prática dos adolescentes das escolas agrícolas da Universidade Federal do Piauí; Escola AnNa Nery Revista de Enfermagem; 13: 4; 863-871.

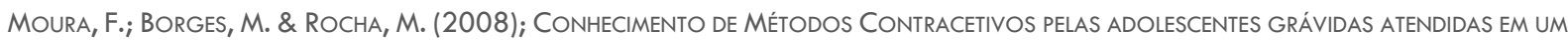
SERVIÇO DE ASSISTÊNCIA PRÉ-NATAL; NÚCLEOS DE PESQUISA DO IMS/EURJ, ISC/UFBA E DA UFRGS.

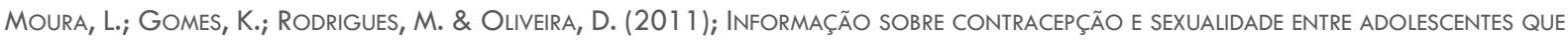
VIVENCIARAM UMA GRAVIDEZ; ACtA PAULISTA De ENFERMAGEM; 24: 3; 320-326.

NorWitz, E. \& Schorge, J. (2003), Compêndio de Obstetrícia e GineCologia; Col. Medicina e Saúde, NN 46; lisboa: Instituto Piaget.

OMS - Organização Mundial de Saúde (1989); Saúde reprodutiva de adolescentes: uma estratéGia para acção; Genebra: OMS.

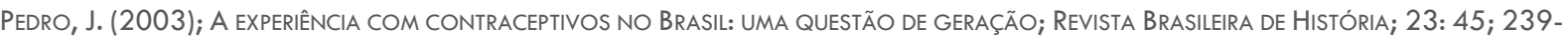
260.

Pestana, M. e Gageiro, J. (2005); Análise de Dados para CiênCias Socials: A complementaridade do SPSS; Lisboa: Edições Sílabo.

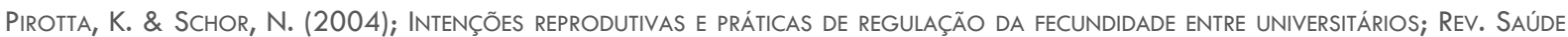
РÚBLICA; 38: 4; 495-502.

RibeIRO, M. \& FERNANDES, A. (2009); COMPORTAMENTOS SEXUAIS de RISCO EM ESTUDANTES DO ENSINO SUPERIOR PÚbliCO DA CIDADE DE Bragança; ReVISta de Psicologia, Saúde \& doenças; 10:1;99-113.

Ribeiro, M. \& Fernandes, A. (2014); CONHECIMENTO E UtILIZAÇÃO dA CONTRACEÇÃo de EMERGÊNCIA EM MULHERES JOVENS ESTUDANTES DO ENSINO SUPERIOR; LIVRO de RESUMOS do IX CONGRESSO NACIONAL DA AsSOCIAÇÃo PORTUGUeSA de LICENCIADOS EM FARMÁCIA, 15-16.

ROQUE, O. (2001); SeMIÓtICA DA CEGONHA: JOVENS, SEXUALIDADE E GRAVIDEZ NÃO DESEJADA; LISBOA: AsSOCIAÇÃo PARA O PLANEAMENTO DA FAMÍLA.

Santos, N.; Rebouças, L.; Boery, R.; Boery, E. \& Silva, S. (2009); Adesão de Universitários ao Uso de Preservativos; Revista SAÚDE.COM; 5: 2; $116-127$.

Vilar, D. (2003); FAlar disso: A eduCação nas famíllas dos Adolescentes; PORTO: EdiçÕes Afrontamento. 